\title{
Saúde, bem-estar e \\ envelhecimento: 0 estudo SABE no \\ Município de São Paulo
}

\section{Health, Well-Being and aging: the SABE Study in São Paulo, Brazil}

Maria Lúcia Lebrão*

Rui Laurenti

Departamento de Epidemiologia, Faculdade de Saúde Pública, Universidade de São Paulo

\section{Resumo}

O projeto SABE (Saúde, bem-estar e envelhecimento) foi coordenado pela Organização Pan-Americana de Saúde com o objetivo de coletar informações sobre as condições de vida dos idosos (60 anos e mais) residentes em áreas urbanas de metrópoles de sete países da América Latina e Caribe - entre elas, o Município de São Paulo - e avaliar diferenciais de coorte, gênero e socioeconômicos com relação ao estado de saúde, acesso e utilização de cuidados de saúde. Por meio de questionário e processo amostral padronizados foram entrevistadas 2.143 pessoas. Encontrou-se que as mulheres são maioria, os imigrantes eram $8,7 \%, 62,6 \%$ viveram por cinco anos ou mais na área rural até os quinze anos de vida. Dos idosos, 13,2\% viviam sós, sendo que esse valor aumentou com a idade e no sexo feminino. Em relação ao estado mental, encontrou-se, pelo Mini Exame do Estado Mental (MEEM), 6,9\% de deterioração cognitiva e 18,1\% de depressão, segundo a Escala de Depressão Geriátrica. As auto-avaliações de saúde mostram que $53,8 \%$ dos entrevistados consideraram a sua saúde regular ou má. Dentre as doenças mais freqüentes estavam a hipertensão (53,3\%); artrite/artrose/reumatismo, 31,7\%; e diabetes, $17,9 \%$. A grande maioria dos idosos não apresentou dificuldades nas atividades básicas de vida diária (80,7\%), e entre aqueles que apresentaram, a maioria tinha dificuldades em uma ou duas atividades. Foram apresentados dados dos arranjos domiciliares encontrados, do acesso e utilização de serviços de saúde, e relativos à renda e condição de trabalho. Conclui-se que as condições de saúde são preocupantes, assim como a insuficiência do sistema de seguridade social.

Palavras-chave: SABE. Envelhecimento. Epidemiologia do envelhecimento.

*Correspondencia: Av. Dr. Arnaldo 715. E-mail: mllebr@usp.br - Projeto financiado pela FAPESP (Processo 99/05125-7). 
Abstract

The SABE project (health, well-being and aging) was coordinated by the Pan American Health Organization and aimed to collect information about the living conditions of the elderly population (aged 60 and older) in urban metropolitan areas in seven Latin American countries, including the city of São Paulo, and to investigate cohort, socioeconomic and gender differences in relation to health status, as well as use and access to heath care. A questionnaire and a standardized sampling process were used to interview 2,143 people. The results show that most of the elderly are women, with a ratio of 142 women to 100 men. $8.7 \%$ were immigrants, and $62.6 \%$ lived for five or more years in the rural area until the age of fifteen. $13.2 \%$ of the elderly lived alone, a percentage that was greater for women and as age increased. For those aged 80 and over, the percentage of living alone was found to be $21.5 \%$; among very old women, that percentage was $17.1 \%$. The Mini Mental State Examination (MMSE) was used to assess cognitive mental functioning. The results show that $6.9 \%$ of the elderly suffered mental impairment. Besides, the older they were, the greater the mental impairment was. However, when the MMSE was associated with the Pfeffer questionnaire, the prevalence dropped to $3.4 \%$. The Geriatric Depression Scale (GDS) revealed that $18.1 \%$ of the elderly suffered from depression and only a small number of them took antidepressants. Their health self assessment data shows that $53.8 \%$ of the interviewees considered their health to be regular or poor, and this assessment differs when variables such as age, sex, years of education, and disability are considered. Among the most frequent illnesses, we found: hypertension (53.3\%) - $80.6 \%$ took some kind of medicine; arthritis/ rheumatism (31.7\%) - 62.6 had some kind of disability, and diabetes (17.9\%). The prevalence of elderly falls was $28.6 \%$. The great majority of the elderly did not have any difficulties in their activities of daily living (ADL) $(80,7 \%)$. Those who had problems usually had difficulties in performing one or two ADLs. Data on their living arrangements and help received according to family support was also presented, and we found that little support was given to ADLs. Income was low - 2.1 minimum wages. We conclude that health status and the inefficiency of the social security system are worrying issues in this elderly population, as a significant number of them are not financially independent and continue to work.

Key Words: SABE. Aging. Epidemiology of aging.

\section{Introdução}

Dentro do processo de envelhecimento do Brasil e da América Latina, é importante conhecer as condições de vida, de saúde, econômicas e de suporte social dos idosos, para que se possa estar preparado para atender às demandas sociais, sanitárias, econômicas e afetivas dessa parcela da população, que, atualmente, é a que mais cresce nessa região.

A população de 60 anos e mais, na América Latina e Caribe, durante o período de 1980 a 2025 terá, em média, dobrado pelo menos uma vez e, em mais da metade dos casos, triplicado antes do ano $2025^{1}$.

Segundo Palloni e Peláez ${ }^{1}$, a importância de se estudar as características do crescimento da população idosa nessa região, prendese ao fato de o cronograma comprimido desse envelhecimento poder ser traçado pela revolução médica e de saúde pública que provocou o declínio da mortalidade há quase meio século. Isso poderá ter implicações na saúde das pessoas que irão alcançar os 60 anos depois de 2000 e que vivenciaram todos os benefícios da tecnologia médica introduzida no período pós-Segunda Guerra mundial, pois os ganhos na sobrevida são mais o resultado da redução à exposição, melhor tratamento e recuperações mais rápidas.

Com esse intuito, a Organização Pan Americana da Saúde, em convênio interagencial integrado pela Comissão Econômica para América Latina e o Caribe (CEPAL), o Fundo de População das Nações Unidas (FNUAP), o Programa de Envelhecimento das Nações Unidas, a Organização Internacional do Trabalho (OIT) e o Banco Interamericano de Desenvolvimento (BID), e com a colaboração de diversos países da Região, desenvolveu, simultaneamente, a Pesquisa SABE (Saúde, bem-estar e envelhecimento) na zona urbana de sete cidades da Região (Bridgetown, Buenos Aires, Havana, México, Montevidéu, Santiago e São Paulo).

\section{Objetivos}

Coletar informações sobre as condições de vida dos idosos (60 anos e mais) residen- 
tes na área urbana do Município de São Paulo e avaliar diferenciais de coorte, gênero e socioeconômicos com relação ao estado de saúde, acesso e utilização de cuidados de saúde.

\section{Metodologia}

O questionário SABE, composto de onze seções que abrangem vários aspectos da vida do idoso, tais como: dados pessoais, avaliação cognitiva, estado de saúde, estado funcional, medicamentos, uso e acesso aos serviços, rede de apoio familiar e social, história laboral e fontes de ingresso, características da moradia, antropometria, flexibilidade e mobilidade (disponível em http:// www.fsp.usp.br/sabe), foi aplicado no ano de 2000 a 2.143 pessoas de 60 anos e mais, residentes no município de São Paulo (Brasil).

Essa amostra é composta por dois segmentos: o primeiro, resultante de sorteio, corresponde à amostra probabilística formada por 1.568 entrevistas. O segundo, formado por 575 residentes nos distritos em que foram realizadas as entrevistas anteriores, corresponde ao acréscimo efetuado para compensar a mortalidade na população de maiores de 75 anos e completar o número desejado de entrevistas nesta faixa etária. Para sorteio de domicílios usou-se o método de amostragem por conglomerados em dois estágios sob o critério de partilha proporcional ao tamanho. Um cadastro permanente de 72 setores censitários, existente no Departamento de Epidemiologia da Faculdade de Saúde Pública, foi considerado a amostra de primeiro estágio. Essa amostra foi tomada do cadastro da Pesquisa Nacional por Amostra de Domicílios (PNAD), 1995, composto por 263 setores censitários sorteados sob o critério de probabilidade proporcional ao número de domicílios. O número mínimo de domicílios sorteados no segundo estágio foi aproximado para 90. A complementação da amostra de pessoas de 75 anos e mais foi realizada através da localização de moradias próximas aos setores selecionados ou, no máximo, dentro dos limites dos distritos aos quais pertenciam os setores sorteados.
Cada questionário teve um peso calculado de acordo com o setor censitário de que faz parte (peso = 1/f) e, para os questionários realizados com indivíduos em domicílios que foram selecionados, porém não sorteados (faixa etária 75 anos e mais), o cálculo do peso foi realizado de acordo com a relação da população de idosos (de ambos os sexos) nessas faixas etárias, residentes no Município de São Paulo em 1998, e o número de idosos nessas mesmas faixas encontradas na amostra final do estudo ${ }^{2}$. A descrição detalhada da metodologia empregada pode ser encontrada no livro "O Projeto SABE no Município de São Paulo: uma abordagem inicial" [disponível em www.opas.org.br/ publicac.cfm] $]^{3}$.

Para a avaliação do estado cognitivo dos idosos, utilizou-se uma versão modificada e validada no Chile, por Icaza e Albala, em 19994, do Mini Exame do Estado Mental (MEEM), desenvolvido por Folstein et $\mathrm{al}^{5}$, que teve o objetivo de reduzir o impacto da escolaridade sobre os resultados. $\mathrm{O}$ resultado no MEEM foi utilizado como filtro para a aplicação do questionário desenvolvido por Pfeffer et $\mathrm{al}^{6}$, o "Pfeffer Functional Activities Questionnaire". Para avaliar sintomas depressivos empregou-se a Escala de Depressão Geriátrica (GDS), desenvolvida por Sheikh e Yesavage?

\section{Resultados e discussão}

\section{Características gerais dos idosos}

Dos 2.143 entrevistados, $58,6 \%$ pertenciam ao sexo feminino, valor bastante próximo ao encontrado no Censo 2000, que foi de $59,5 \%$. Esse dado chama a atenção para a feminização da velhice, pois a razão de feminilidade em São Paulo foi de 142, ou seja, para cada 100 homens havia 142 mulheres. Embora esse valor seja elevado, em algumas das cidades estudadas ele foi mais alto ainda, como em Buenos Aires (162), Montevidéu (176) ou Santiago (192).

As idades variaram entre 60 e 100 anos, com média de 68 anos, uma das mais baixas das sete localidades, só perdendo para o Méxi- 
co, com 63 anos de média. Em 1989, quando Ramos et $\mathrm{al}^{8}$ realizaram inquérito domiciliar entre idosos em algumas regiões do Município de São Paulo, essa média era de 69 anos.

Os imigrantes eram em número significativo, refletindo, ainda, os movimentos migratórios do início dos anos 1900, pois 8,7\% não nasceram no Brasil, sendo, dentre eles, $38,7 \%$ procedentes de Portugal, 16,8\%, do Japão, $16,5 \%$ da Itália e $11 \%$ da Espanha, além de outros.

Era grande o número de idosos que viveram, por cinco anos e mais, na zona rural até os 15 anos de idade: $62,6 \%$. Isso pode ter um significado importante, uma vez que essas pessoas podem ter sido privadas de escola, atenção à saúde e outros benefícios que a proximidade da zona urbana pode trazer. Isso é corroborado pelo fato de $21,7 \%$ não saberem ler ou escrever um bilhete. No estudo de $\operatorname{Ramos}^{8}$ esse valor foi de $35 \%$, o que mostra a melhoria no nível de escolaridade dos nossos idosos, embora nesse quesito São Paulo perca fortemente para as outras capitais: Bridgetown tinha apenas $0,8 \%$ da sua população idosa sem escolaridade, Buenos Aires, 3,3 e Montevidéu, 4,2\%.

A maioria dos idosos vivia acompanhada - $86,8 \%$ - porém esse valor cai à medida que eles envelhecem, pois entre aqueles com 80 anos e mais, $21,5 \%$ moram sozinhos. A se pensar na população de pessoas de 80 anos e mais na cidade de São Paulo em 2000 , terse-á 25.000 muito idosos vivendo sós. Aqui, o gênero traz uma diferença acentuada, pois entre as mulheres esse número se eleva para $25,3 \%$, enquanto entre os homens ele é de apenas 13,8\%. Se essa condição já inspira atenção entre os idosos em geral, na velhice avançada isso é motivo de um olhar diferenciado para essa população. Sabidamente, os homens recasam em muito maior proporção do que as mulheres; daí, essa diferença. As cifras comprovam: enquanto as viúvas eram $42,6 \%$, os viúvos eram $11,1 \%$.

\section{Declínio cognitivo e depressão}

Quanto à avaliação do estado mental, obteve-se neste estudo, segundo os resulta- dos do MEEM, uma prevalência de deterioração cognitiva de $6,9 \%$, sendo de $4,2 \%$ para as pessoas na faixa etária de 60 a 74 anos e de $17,7 \%$ para aquelas com 75 anos ou mais. Analisando-se as porcentagens de pessoas que obtiveram pontuação igual ou inferior a 12 no MEEM $^{4}$, verificou-se um aumento progressivo dessas com o avançar da idade (Tabela 1).

Tabela 1 - Distribuição de freqüência (\%) dos idosos, segundo idade e resultado do MEEM, São Paulo, 2000.

Table 1 - Frequency distribution (\%) of the elderly, by age and MMSE result, São Paulo, 2000.

\begin{tabular}{lcc}
\hline Idade & \multicolumn{2}{c}{ MEEM } \\
& $\begin{array}{c}13 \text { ou }+ \\
\%\end{array}$ & $\begin{array}{c}<\text { ou }=12 \\
\%\end{array}$ \\
\hline $60-64$ & 96,4 & 3,6 \\
$65-69$ & 96,7 & 3,3 \\
$70-74$ & 93,5 & 6,5 \\
$75-79$ & 87,2 & 12,8 \\
$80-84$ & 80,5 & 19,4 \\
85 e + & 68,4 & 31,6 \\
Total & 93,1 & 6,9 \\
\hline
\end{tabular}

Segundo Cerqueira ${ }^{9}$, essa progressão assemelha-se à de dados previamente relatados na literatura internacional e brasileira.

Combinando-se o MEEM com o Questionário Pfeffer, as taxas de prevalência de declínio cognitivo diminuem acentuadamente - de 6,9\%, só com o MEEM, para 3,4\% com o MEEM + Pfeffer - podendo indicar que este consegue dar maior especificidade para a medida de deterioração cognitiva mais grave, especialmente após os 75 anos $^{9}$.

Em relação à depressão, verificou-se uma prevalência de $18,1 \%$ de sintomas depressivos, com um diferencial importante entre homens e mulheres $(12,7 / 22,0 \%$, respectivamente). Constatou-se, ainda, que foi mais alta a prevalência de sintomas depressivos entre as pessoas com 60 a 64 anos de idade (19,5\%) atingindo $13 \%$ a prevalência para aqueles com 75 ou mais anos de idade.

Estudos citados por Cerqueira ${ }^{9}$ apontam que, apesar da constatada sobrecarga, in- 
clusive econômica, da depressão, ela ainda é pouco diagnosticada, e que, apesar dos transtornos depressivos serem crônicos, são tratáveis. Dados da presente investigação, no entanto, demonstram que apenas uma pequena parcela dos idosos identificados como tendo sintomas depressivos, leves ou graves, referiu o uso de medicação antidepressiva (Tabela 2).

\section{Condições de saúde}

As avaliações de saúde auto-referidas hoje não são mais consideradas meramente impressões relacionadas a condições reais de saúde. Muitos estudos recentes têm mostrado que os indivíduos que relatam condições de saúde escassa ou pobre têm riscos de mortalidade consistentemente mais altos que aqueles que reportam melhor estado de saúde ${ }^{10}$.

Nesta pesquisa, à pergunta de como o entrevistado considerava a sua saúde - excelente, muito boa, boa, regular ou má - obteve-se o resultado de 53,8\% para regular ou má e $46,2 \%$ para excelente, muito boa ou boa.

Esses números, no entanto, variam de acordo com o sexo e a idade do entrevistado.
Ao aumentar a idade, a auto-avaliação, quanto a ser muito boa ou boa, cai para $43,8 \%$ entre aqueles que têm 75 anos e mais. Entre os sexos, a mulher tem pior auto-avaliação, sendo entre as de 75 anos e mais, a mais baixa $42,9 \%$ de muito boa ou boa (Tabela 3 ).

Outras condições podem alterar a percepção das pessoas em relação à sua saúde. A escolaridade é uma delas, pois os idosos sem escolaridade têm uma avaliação de regular ou má 1,2 vezes maior do que aqueles com 7 a 12 anos de estudo $(65,7 \% / 29,2 \%$, respectivamente) e a mulher sem escolaridade sobrepassa o homem, nas mesmas condições, em $6,7 \%(68,1 \% / 61,4 \%$, respectivamente).

Viver só favorece a auto-avaliação, pois dos que estão nessa condição, 51,8\% consideraram a sua saúde muito boa ou boa. Essa melhor avaliação se repete em todas as faixas etárias exceto entre os homens de 75 anos e mais onde essa situação se inverte, caindo para $44,8 \%$. Isso pode ocorrer pelo fato de aqueles que vivem sós realmente estarem em melhores condições.

Por outro lado, a presença de incapacidade parece ser um fator determinante na autoavaliação de saúde. Ter, pelo menos, uma in-

Tabela 2 - Grau de depressão (medido pela GDS) segundo sexo e o uso de antidepressivos, São Paulo, 2000.

Table 2 - Level of depression (measured by GDS), by sex and use of antidepressants, São Paulo, 2000.

\begin{tabular}{|c|c|c|c|c|}
\hline \multirow{3}{*}{ Depressão } & \multicolumn{4}{|c|}{ Antidepressivos } \\
\hline & \multicolumn{2}{|c|}{ Não Usa } & \multicolumn{2}{|c|}{ Usa } \\
\hline & Homens & Mulheres & Homens & Mulheres \\
\hline Normal & 98,7 & 96,2 & 1,3 & 3,8 \\
\hline Leve & 97,3 & 90,7 & 3,7 & 9,3 \\
\hline Grave & 90,0 & 88,6 & 10,0 & 11,4 \\
\hline
\end{tabular}

Tabela 3 - Auto-avaliação de saúde segundo sexo e idade, São Paulo, 2000.

Table 3 - Health self-assessment, by gender and age, São Paulo, 2000.

\begin{tabular}{lccccccc}
\hline Auto-avaliação & \multicolumn{2}{c}{ Homens } & Sub-Total & \multicolumn{2}{c}{ Mulheres } & Sub-Total & Total \\
& 60 a 74 & $75 \mathrm{e}+$ & & 60 a 74 & $75 \mathrm{e}+$ & & \\
\hline Muito boa/boa & 49,6 & 43,8 & 48,5 & 44,7 & 42,9 & 44,3 & 46,0 \\
Regular/má & 50,4 & 56,2 & 51,5 & 55,0 & 57,0 & 55,4 & 54,0 \\
Total & 100,0 & 100,0 & 100,0 & 100,0 & 100,0 & 100,0 & 100,0 \\
\hline
\end{tabular}


capacidade para as atividades básicas de vida diária, reduz à metade a disposição de considerar a sua saúde muito boa ou boa: $23,8 \%$.

A Tabela 4 mostra a distribuição das doenças incluídas no questionário. A doença de maior prevalência foi a hipertensão, com uma freqüência relativamente alta, levandose em conta que se trata de doença autoreferida, ou seja, dependente de utilização de serviços de saúde e conseqüente conhecimento da sua existência.

Observou-se que apenas $80,6 \%$ dos hipertensos tomavam medicamentos para controlar a pressão, sendo $84,8 \%$ das mulhe- res e 73,5\% dos homens hipertensos. Apenas $45,1 \%$ dos hipertensos informaram que nos últimos 12 meses perderam peso intencionalmente ou seguiram uma dieta especial.

Vários estudos longitudinais e transversais confirmam que, mesmo nos idosos, a hipertensão sistólica, por si ou combinada com a pressão diastólica elevada, continua sendo um importante fator preditivo da mortalidade e morbidade cardiovasculares. Nos idosos, a incidência de doenças cardiovasculares se relaciona mais estreitamente com a pressão sistólica do que com a diastólica e não há dúvidas de que a pressão

Tabela 4 - Respostas positivas (\%) à pergunta sobre doenças crônicas segundo idade e sexo, São Paulo, 2000.

Table 4 - Affirmative answers (\%) to the question about chronic diseases, by age and gender, São Paulo, 2000.

\begin{tabular}{|c|c|c|c|}
\hline DOENÇA CRÔNICA & $60-74$ & 75 e + & TOTAL \\
\hline Pressão Alta & 52,8 & 55,1 & 53,3 \\
\hline Fem & 55,1 & 60,2 & 56,3 \\
\hline Masc & 49,8 & 45,9 & 49,1 \\
\hline Artrite/Artrose/Reumat. & 30,5 & 36,0 & 31,7 \\
\hline Fem. & 38,5 & 43,3 & 39,6 \\
\hline Masc. & 20,0 & 23,2 & 20,6 \\
\hline Problema cardíaco & 18,2 & 24,3 & 19,5 \\
\hline Fem. & 17,2 & 24,0 & 18,8 \\
\hline Masc. & 19,5 & 24,7 & 20,5 \\
\hline Diabetes & 18,3 & 16,3 & 17,9 \\
\hline Fem & 19,3 & 17,0 & 18,7 \\
\hline Masc. & 17,2 & 14,9 & 16,8 \\
\hline Osteoporose & 13,4 & 16,9 & 14,2 \\
\hline Fem. & 21,8 & 23,88 & 22,3 \\
\hline Masc. & 2,4 & 4,32 & 2,7 \\
\hline D. Crônica Pulmonar & 12,0 & 12,7 & 12,2 \\
\hline Fem. & 10,7 & 10,7 & 10,7 \\
\hline Masc. & 13,8 & 16,4 & 14,3 \\
\hline Embolia/Derrame & 6,9 & 8,2 & 7,2 \\
\hline Fem. & 5,7 & 6,9 & 6,0 \\
\hline Masc. & 8,6 & 10,5 & 8,9 \\
\hline Tumor Maligno & 2,9 & 4,6 & 3,3 \\
\hline Fem. & 3,0 & 5,0 & 3,5 \\
\hline Masc. & 2,9 & 4,0 & 3,1 \\
\hline
\end{tabular}


arterial elevada é o fator de risco mais importante para os acidentes vasculares cerebrais, isquêmicos ou hemorrágicos ${ }^{11}$.

Segue-se à hipertensão, quanto à magnitude da freqüência, a prevalência da artrite/ reumatismo/artrose, com $31,7 \%$, predominando bastante no sexo feminino - 39,6\%, contra $20,6 \%$ entre os homens. Ao juntar essas três condições, a pesquisa pretendeu identificar a presença de dores articulares, muitas vezes incapacitantes, o que vem ao encontro do descrito por Santos ${ }^{12}$, onde se mostra que, embora tendo maior sobrevida, a mulher tem uma proporção menor dessa, sem incapacidade.

Difícil identificar em morbidade referida tratar-se de artrite, artrose ou algum outro tipo específico de reumatismo. Infere-se sua existência por meio de queixas como dores articulares, limitação de atividades ou, particularmente neste inquérito, pelo fato de ter sido um profissional de saúde a fazer o diagnóstico. Em nosso meio, o leigo geralmente refere como tendo "reumatismo" ou, algumas vezes, "artrite".

A limitação de atividades é bastante importante no idoso e o conjunto "artrite/reumatismo/artrose” é um dos principais responsáveis por esse quadro. Neste estudo, $22,1 \%$ informaram ter muita limitação e $40,5 \%$ pouca limitação, somando $62,6 \%$ com algum tipo de limitação, dentre aqueles que referiram "artrite/reumatismo/artrose".

Outro dos indicadores de morbidade bastante utilizados em geriatria é a prevalência de diabetes mellitus. Essa condição foi referida em 17,9\% da amostra, com ligeiro predomínio no sexo feminino. Dos que informaram ter diabetes mellitus, $64,3 \%$ referiram controle com medicação oral e $12,7 \%$ o faziam com insulinoterapia. Em relação ao controle dessa doença, deve-se ressaltar o fato de que $20,2 \%$ deles não a têm controlada ou não sabem referir. Acrescese a esse fato que o diabetes foi a única doença crônica cuja freqüência diminui com o avançar da idade, muito provavelmente pelos óbitos decorrentes de suas complicações.

As complicações circulatórias relativas aos acidentes vasculares cerebrais foram investigadas por meio da pergunta "teve uma embolia, derrame, ataque, isquemia ou trombose cerebral?". Em nosso meio, o termo mais usado e conhecido pelo leigo é "derrame" e é sempre facilmente identificável na morbidade referida.

Foi encontrado que $7,2 \%$ dos inquiridos responderam afirmativamente, sendo um pouco maior naqueles com 75 anos e mais: $8,2 \%$, contra $6,9 \%$ dos 60 aos 74 anos. A freqüência também foi um pouco maior nos homens $(8,9 \%)$ do que nas mulheres $(6,0 \%)$, e em ambos os sexos aumenta com a idade, chegando nos homens a ser $10,5 \%$, acima dos 75 anos (na mulher, neste grupo etário, a freqüência foi $6,9 \%$ ). Uma das complicações mais freqüentes e, porque não dizer, mais temidas, dos acidentes vasculares cerebrais são as seqüelas (hemiplegia, dificuldade da fala e outros) e, neste estudo, encontrou-se que $75,3 \%$ dos que tiveram "derrame" apresentaram-nas, com valor um pouco maior no homem $(78,2 \%)$ do que na mulher $(72,4 \%)$.

A freqüência referida de "derrame" é relativamente baixa quando comparada à hipertensão arterial, diabetes e problemas cardíacos. Isso é compreensível tendo-se em vista que a alta letalidade nessa condição é apenas para os acidentes vasculares hemorrágicos, sendo muito menor para as embolias e tromboses, e estes, na prática, são muito mais freqüentes, levando assim a uma maior sobrevida para o conjunto dos derrames.

Além das doenças, outros agravos tais como as quedas constituem uma importante ocorrência nos idosos sendo que elas têm como principal conseqüência as fraturas, em particular do fêmur (colo ou outras partes). As fraturas nos idosos podem levar a vários tipos de complicações, particularmente póscirúrgicas, e têm como conseqüência mais grave, a morte.

Verificou-se que 28,6\% dos idosos referiram "quedas", sendo que essa freqüência aumentou com a idade: $26,2 \%$ naqueles com 60 a 74 anos e $36,9 \%$ acima de 75 anos. É interessante $o$ fato de que as quedas são mais freqüentes nas mulheres $(33,0 \%)$ do que nos 
homens (22,3\%). Possivelmente, essa maior freqüência nas mulheres seria um indicativo de, nesse sexo, ocorrer mais a fratura anterior à queda, visto que em $22,3 \%$ delas o médico informou que tinham osteoporose, o que ocorreu em apenas $2,7 \%$ dos homens. $\mathrm{Na}$ amostra estudada, a freqüência de fraturas foi pequena $-0,4 \%$ e $0,7 \%$, respectivamente - informadas como do quadril e do pulso.

\section{Saúde bucal}

Em relação à saúde bucal dos idosos, menos de $1 \%$ deles tem todos os dentes. A essa medida da mutilação dentária não corresponde, entretanto, uma autopercepção negativa das condições bucais. Embora as dificuldades para mastigar tenham sido mencionadas por $22 \%$ dos idosos e $3 \%$ tenham admitido dificuldades para engolir bem, o amplo emprego de vários tipos de próteses dentárias parece contribuir para minimizar os efeitos da mutilação e para uma autopercepção positiva ${ }^{13}$.

Com efeito, $86 \%$ dos idosos que perderam mais da metade dos dentes utilizam algum tipo de prótese. Assim, o acesso aos serviços odontológicos no município de São Paulo parece ser suficientemente amplo, para atendimentos básicos, a ponto de os serviços produzidos terem impacto significativo na autopercepção das condições de saúde bucal dos usuários. Ainda que se possa questionar a prática mutiladora e, essencialmente, reabilitadora dessas ações odontológicas, é preciso considerar que, apenas, $12 \%$ dos idosos declararam-se insatisfeitos com a imagem dos seus dentes no espelho, e 92\% afirmaram que nunca deixaram de sair à rua ou conversar por causa de suas condições dentárias ${ }^{13}$.

\section{Avaliação nutricional}

A questão nutricional das pessoas entrevistadas foi verificada através do Índice de Massa Corporal (IMC), mesmo que a classificação do estado nutricional de idosos segundo esse indicador seja ainda bastante dis- cutida, havendo divergência, entre vários estudos, quanto aos valores empregados ${ }^{14}$.

A maior proporção de sobrepeso e obesidade verificada para o sexo feminino $(40,5 \%)$ em comparação ao masculino $(21,7 \%)$, parece refletir maior quantidade (proporcional) de gordura corporal, pois as mulheres apresentam valores de massa corporal e estatura inferiores aos homens. Sabese que isso ocorre em todas as idades e se dá, principalmente devido à gordura essencial, que no sexo feminino é, aproximadamente, quatro vezes superior à dos indivíduos do sexo masculino ${ }^{14}$.

A quase totalidade dos idosos se considerou bem nutrida (91,6\%); no entanto, somente $67,4 \%$ disseram fazer três ou mais refeições completas ao dia (incluído aí o desjejum).

\section{Atividades de vida diária}

A funcionalidade dos idosos foi avaliada a partir do seu desempenho referido nas atividades cotidianas que foram subdivididas em dois grandes grupos: Atividades Básicas de Vida Diária (ABVDs) e Atividades Instrumentais de Vida Diária (AIVDs).

As ABVDs relacionam-se ao autocuidado e o seu comprometimento implica, geralmente, na necessidade de auxílio de terceiros, o que as torna mais onerosas e mais complexas. A Tabela 5 mostra a proporção de idosos que referiram dificuldades no desempenho das ABVDs.

Observa-se que a maioria dos idosos, $80,7 \%$, não apresenta limitações funcionais que impeçam o seu auto-cuidado. Porém, $19,2 \%$ deles as apresentam, representando para o Município de São Paulo, hoje, a cifra de aproximadamente 200.000 pessoas cujas limitações estão relacionadas à sobrevivência e boa qualidade de vida.

Entre os idosos que referiram dificuldades, $70,5 \%$ o fizeram em uma ou duas atividades e 29,0\% em três ou mais. Quando essa análise é estratificada por faixas etárias e sexo observa-se, entre os idosos em idade avançada (75 anos e mais), uma queda no desempenho e, conseqüente aumento no grau 
Tabela 5 - Proporção (\%) dos idosos que apresentaram dificuldades no desempenho das ABVDs segundo idade e sexo, São Paulo, 2000

Table 5 - Percentage of elderly who had difficulties in performing ADLs, by age and gender, São Paulo, 2000.

\begin{tabular}{lccccccc}
\hline $\begin{array}{l}\text { ABVDs com } \\
\text { dificuldades }\end{array}$ & $60-74$ anos & $\begin{array}{c}\text { Feminino } \\
75 \text { anos }+\end{array}$ & Total & $60-74$ anos & $\begin{array}{c}\text { Masculino } \\
75 \text { anos }+\end{array}$ & Total & TOTAL \\
\hline Nenhuma & 81,7 & 64,6 & 77,5 & 88,0 & 73,1 & 85,2 & 80,7 \\
$1-2$ & 14,5 & 21,3 & 16,1 & 9,0 & 14,5 & 10,0 & 13,6 \\
3 e + & 3,8 & 13,7 & 6,2 & 3,0 & 12,4 & 4,8 & 5,6 \\
N R & - & 0,4 & 0,2 & - & - & - & 0,1 \\
TOTAL & 100,0 & 100,0 & 100,0 & 100,0 & 100,0 & 100,0 & 100,0 \\
\hline
\end{tabular}

de limitações funcionais, mais acentuados entre os homens, com maior grau de dependência (Tabela 6).

Analisadas separadamente, as atividades de vestir-se, mobilização e banho apresentam os maiores índices de dificuldades, sendo que, com exceção da atividade "alimentação", nas demais as mulheres referem maior comprometimento. Quanto à análise da faixa etária, reitera-se o já observado, colocando os idosos em velhice avançada em uma situação de maior vulnerabilidade e, conseqüentemente, de maior risco.

A análise da ajuda recebida para o desempenho das atividades básicas mostra um desequilíbrio entre as necessidades referidas e o auxílio efetivamente recebido. Em nenhuma das dificuldades referidas, a ajuda oferecida aproximou-se de $100 \%$. A atividade relacionada à alimentação apresentou a maior proporção de auxílio recebido (em torno de $70 \%$ ) quando comparada com as outras atividades, provavelmente, por essa estar diretamente ligada à questão de sobrevivência do idoso. As outras atividades rela- cionadas a bem-estar e conforto e, conseqüentemente, a uma boa qualidade de vida, mostram-se muito aquém das demandas referidas. As mulheres, em geral e nas idades de 60 a 74 anos, recebem menos auxílio que os homens. Pode-se inferir uma provável influência do estado marital, pois entre elas há um maior percentual de ausência de companheiro do que entre os homens, sendo bem conhecido o auxílio prestado pelas companheiras. Esses percentuais acabam se tornando semelhantes na velhice avançada em ambos os sexos.

As AIVDs relacionam-se com a participação efetiva do idoso na comunidade, onde a presença de dificuldades implica mais em uma redistribuição de tarefas entre os membros familiares (como alguém para ir fazer compras ou auxiliar nas tarefas domésticas) e menos em cuidado direto. Observa-se que a maioria dos idosos não apresenta dificuldades (Tabela 7), porém isso ocorre em menor proporção quando comparado com as ABVDs. Essa não é apenas uma peculiaridade dos idosos de São Paulo, pois nos outros

Tabela 6 - Proporção (\%) dos idosos com dificuldades no desempenho de ABVDs segundo número de atividades, grupo etário e sexo, São Paulo, 2000.

Table 6 - Percentage of elderly with difficulties in performing ADLs, by number of activities, age group and gender. São Paulo, 2000.

\begin{tabular}{lccccccc}
\hline $\begin{array}{l}\text { ABVDs com } \\
\text { dificuldades }\end{array}$ & $60-74$ anos & $\begin{array}{c}\text { Feminino } \\
75 \text { anos e }+\end{array}$ & Total & $60-74$ anos & $\begin{array}{c}\text { Masculino } \\
75 \text { anos e }+\end{array}$ & Total & TOTAL \\
\hline $1-2$ & 78,8 & 60,3 & 71,8 & 74,8 & 54,0 & 67,6 & 70,5 \\
3 e + & 20,5 & 38,7 & 27,4 & 25,2 & 46,0 & 32,4 & 29,0 \\
Não respondeu & 0,7 & 1,0 & 0,8 & - & - & - & 0,5 \\
TOTAL & 100,0 & 100,0 & 100,0 & 100,0 & 100,0 & 100,0 & 100,0 \\
\hline
\end{tabular}


países do estudo SABE essa informação se repete $^{15}$. Esses dados reforçam a já conhecida relação hierárquica de perda de habilidades funcionais que ocorre fisiologicamente no envelhecimento e que se direciona, normalmente, das AIVDs para as $\mathrm{ABVDs}^{8}$. Assim, observa-se que $26,5 \%$ dos idosos apresentam dificuldades no desempenho das AIVDs, necessitando, portanto, de auxílio de outras pessoas que atuem como elo de ligação entre eles e seu entorno social. Isso é mais acentuado entre as mulheres de ambas as faixas etárias analisadas e pioram expressivamente para ambos os sexos na velhice avançada, contribuindo para o afastamento do idoso do convívio social e conseqüente tendência ao isolamento ${ }^{16}$.

Muitos dos estudos que traçam o perfil dos idosos trabalham com as atividades de vida diária em conjunto, somando as dificuldades de desempenho de ambas, atividades básicas e instrumentais. Neste estudo procurou-se enfocar separadamente os dois conjuntos de atividades, uma vez que o tipo de ajuda necessária, as demandas de serviços e os custos decorrentes são muito diferentes. Outra questão a considerar são as atividades avaliadas. Foi utilizada a escala de Lawton com as oito atividades avaliadas. Em outros estudos, o número de atividades utilizadas foi em menor número, o que faz com que os resultados sejam diferentes, requerendo cuidado nas comparações a serem feitas.

\section{Suporte familiar}

Buscando conhecer a rede de suporte familiar apresentada pelos idosos fez-se ini- cialmente uma relação descritiva das várias combinações de co-residentes dos idosos, resultando em 65 arranjos familiares diferentes para o Município de São Paulo. Essa mesma análise estendida aos outros países participantes do Projeto SABE mostra que o número de arranjos oscila entre $46 \mathrm{em}$ Buenos Aires (Argentina), 56 em Montevidéu (Uruguai), 64 em Bridgetown (Barbados), 67 em Havana (Cuba), 76 em Santiago (Chile) e 77 na Cidade do México (México).

Quando esses arranjos familiares foram ordenados por freqüência, verificou-se que a maioria dos idosos vive com sua família de procriação (união de duas pessoas de sexos diferentes que darão origem a uma descendência comum) reforçando a importância dessa no cuidado dos mesmos. Cabe ressaltar que, na maioria das vezes, o cônjuge também é idoso ou muito próximo dessa faixa etária e o seu potencial de auxílio pode estar limitado. Outro dado preocupante foi o contingente de idosos vivendo sós, pois esse arranjo ocupou o quarto posto. Dada a maior probabilidade de recasamento entre os homens, a condição de viver só ocorre com maior freqüência entre as mulheres $(7,5 \% \mathrm{e}$ $17,0 \%$ respectivamente).

Embora a classificação das famílias difira conforme a fonte consultada ${ }^{17-19}$, foi objetivo desse estudo apresentar os arranjos aparentemente mais vulneráveis, de forma a propiciar uma reorganização assistencial mais efetiva. $\mathrm{O}$ agrupamento ora proposto foi subdividido basicamente em relações intrageracionais (entre iguais, ou seja, entre idosos) $\mathrm{e}$ intergeracionais (considerando duas, três ou

Tabela 7 - Proporção (\%) dos idosos que apresentaram dificuldades no desempenho das AIVDs segundo grupo etário e sexo, São Paulo, 2000.

Table 7 - Percentage of elderly who had difficulties in performing IADLs, by age group and gender. São Paulo, 2000.

\begin{tabular}{lccccccc}
\hline $\begin{array}{l}\text { AlVDs com } \\
\text { dificuldades }\end{array}$ & $60-74$ anos & $\begin{array}{c}\text { Feminino } \\
75 \text { anos e }+\end{array}$ & Total & $60-74$ anos & $\begin{array}{c}\text { Masculino } \\
75 \text { anos e }+\end{array}$ & Total & TOTAL \\
\hline Nenhuma & 75,6 & 41,5 & 67,4 & 87,1 & 58,8 & 81,6 & 73,3 \\
$1-2$ & 16,8 & 31,2 & 20,3 & 6,4 & 19,0 & 8,8 & 15,5 \\
3 e + & 7,3 & 26,8 & 12,0 & 6,5 & 22,1 & 9,5 & 11,0 \\
Não respondeu & 0,3 & 0,5 & 0,3 & - & 0,1 & 0,1 & 0,2 \\
TOTAL & 100,0 & 100,0 & 100,0 & 100,0 & 100,0 & 100,0 & 100,0 \\
\hline
\end{tabular}


mais gerações co-residentes) ${ }^{20}$. Os arranjos encontrados foram assim subdivididos: Grupo $A$ (vive só), Grupo B (somente com o cônjuge), Grupo $C$ (Cônjuge + filho(s), Cônjuge + filho(s) +genro/nora, somente com filho(s)), Grupo D (Arranjos trigeracionais), Grupo $E$ (Arranjos intrageracionais), Grupo F (Arranjos intrageracionais + neto (sem filhos)) e Grupo $\boldsymbol{G}$ (incluindo não familiares) ${ }^{16}$.

No Município de São Paulo, os idosos coresidem com cônjuge e/ou filhos (sem netos) em primeiro lugar; em segundo lugar, somente com cônjuges; em terceiro, em arranjos trigeracionais e em quarto, sozinhos (Tabela 8). Esses arranjos diferem quando analisados quanto ao sexo: as mulheres moram, proporcionalmente, mais sós que os homens e esses co-residem mais com cônjuge e/ou filho(s) ou somente com cônjuge, demonstrando um maior potencial de ajuda para os homens que para as mulheres. As idosas, por sua vez, são mais acolhidas em arranjos trigeracionais, talvez por poderem colaborar nas funções familiares como, por exemplo, o cuidado dos netos. Co-residir com cuidador contratado ocupou a última posição, demonstrando ser a família o principal recurso disponível para atender às demandas dos idosos ${ }^{16}$.

Quando o auxílio recebido é analisado por tipo de arranjo familiar existente (Tabela 9), observa-se que o arranjo menos continente é o que convive apenas com neto(s) (Grupo F) considerando que os idosos que vivem sós (Grupo A) não contam com qual- quer auxílio de co-residentes, dado que eles não existem. Os arranjos que contam, prioritariamente, com pessoas idosas, também não respondem satisfatoriamente às necessidades dos mesmos, ou seja, $69,4 \%$ para os arranjos intrageracionais (Grupo E) e $64 \%$ para os que co-residem apenas com o cônjuge (Grupo B). Os arranjos familiares mais continentes são os bi ou trigeracionais, onde, respectivamente, $59,5 \%$ e $47,9 \%$ dos idosos referiram não receber ajuda. Esses estão associados aos núcleos familiares maiores (famílias trigeracionais) e com a presença de outros cuidadores, corroborando o encontrado por Saad ${ }^{18}$, onde a co-residência mostrou-se mais significativa que o número de filhos existente. Mesmo nessas circunstâncias, a ajuda fornecida está apenas ao redor de $50 \%$, sendo a mais importante advinda de arranjos com não familiares, onde provavelmente existem cuidadores contratados para esse fim. Nesses, 43,6\% dos idosos referem não receber ajuda, o menor percentual encontrado no estudo.

\section{Uso de medicamentos e serviços de saúde}

A grande maioria dos idosos estava tomando algum medicamento no momento da entrevista $(86,7 \%)$, sendo que, destes, $86,0 \%$ tinham prescrição médica e $10,1 \%$ se automedicavam. A aquisição desses medicamentos é problemática, pois $71,7 \%$ pagavam do seu próprio bolso, razão pela qual muitos interromperam a medicação no últi-

Tabela 8 - Proporção (\%) de idosos segundo arranjos familiares (reagrupamento), sexo do entrevistado e postos, São Paulo, 2000.

Table 8 - Percentage of elderly by living arrangement, gender and position, São Paulo, 2000.

\begin{tabular}{cccc}
\hline $\begin{array}{c}\text { ARRANJOS } \\
\text { FAMILIARES }\end{array}$ & Feminino & SEXO & TOTAL \\
\hline A & $17,1\left(3^{\circ}\right)$ & Masculino & \\
B & $15,6\left(4^{\circ}\right)$ & $7,5\left(4^{\circ}\right)$ & $13,1\left(4^{\circ}\right)$ \\
C & $30,1\left(1^{\circ}\right)$ & $26,2\left(2^{\circ}\right)$ & $20,0\left(2^{\circ}\right)$ \\
D & $21,1\left(2^{\circ}\right)$ & $35,9\left(1^{\circ}\right)$ & $32,5\left(1^{\circ}\right)$ \\
E & $6,2\left(5^{\circ}\right)$ & $15,8\left(3^{\circ}\right)$ & $18,9\left(3^{\circ}\right)$ \\
F & $5,4\left(6^{\circ}\right)$ & $7,3\left(5^{\circ}\right)$ & $6,7\left(5^{\circ}\right)$ \\
G & $4,6\left(7^{\circ}\right)$ & $3,3\left(7^{\circ}\right)$ & $\left.4,5^{\circ}\right)$ \\
TOTAL & 100,0 & $3,9\left(6^{\circ}\right)$ & $4,3\left(7^{\circ}\right)$ \\
\hline
\end{tabular}


Tabela 9 - Proporção (\%) de idosos segundo ajuda recebida, arranjos familiares e número de dificuldades, São Paulo, 2000.

Table 9 - Percentage of elderly, by help received, living arrangement and number of difficulties, São Paulo, 2000.

\begin{tabular}{ccccc}
\hline $\begin{array}{c}\text { ARRANJOS } \\
\text { FAMILIARES }\end{array}$ & $\begin{array}{c}\text { Ajuda recebida por dificuldades apresentadas } \\
\text { Não recebe } \\
\text { nenhuma ajuda }\end{array}$ & $\begin{array}{c}\text { necebe ajuda em } \\
\text { 1 ou 2 ABVDs }\end{array}$ & $\begin{array}{c}\text { ABVi* } \\
\text { Recebe ajuda em } \\
\text { 3 ou + ABVDs }\end{array}$ & TOTAL \\
\hline A & 83,6 & 14,5 & 1,9 & 100,0 \\
B & 64,0 & 17,8 & 18,2 & 100,0 \\
C & 59,4 & 29,2 & 11,4 & 100,0 \\
D & 47,9 & 28,5 & 23,6 & 100,0 \\
E & 69,4 & 24,6 & 6,0 & 100,0 \\
F & 82,6 & 4,2 & 13,2 & 100,0 \\
G & 43,6 & 29,8 & 26,6 & 100,0 \\
TOTAL & 60,6 & 24,1 & 15,3 & 100,0 \\
\hline
\end{tabular}

*Refere-se à ajuda recebida, independente do número de dificuldades apresentadas. Nessa tabela encontram-se apenas os idosos que referiram dificuldade no desempenho das ABVDs.

mo mês (16,8\%), sendo que $54,0 \%$ destes por questão de custo. Esse fato é altamente preocupante considerando-se os problemas que pode gerar, tais como piora no controle das doenças crônicas e conseqüente aumento das incapacidades.

A maioria dos entrevistados, cerca de $60 \%$, informou estar vinculado ao Sistema Único de Saúde. Embora o SUS ofereça cobertura universal, um número expressivo de entrevistados não fez menção a ele, talvez pelo fato de não utilizá-lo. A referência ao SUS diminuiu com o aumento dos anos de escolaridade, passando para $42 \%$ para o segundo grupo de escolaridade, 5 a 8 anos; $19,7 \%$ para o grupo de 9 a 11 anos e $15 \%$ para o grupo de $12 \mathrm{ou}$ mais anos de escolaridade ${ }^{21}$.

O Plano de Saúde Privado foi a segunda forma de seguro de saúde mais citada, sendo que a sua cobertura aumentou com a escolaridade, passando de $28,6 \%$ no primeiro grupo para $49,7 \%$ no segundo, $64,5 \%$ no terceiro e $61,7 \%$ no último grupo, o de escolaridade mais alta. A proporção ligeiramente menor de cobertura pelo Plano de Saúde Privado, no grupo de escolaridade mais alta, deve decorrer de este grupo ser o que fez maior uso de "Outro Seguro Público de Saúde", com $26,1 \%$, cuja cobertura foi de $7,5 \%$ para os entrevistados com até 4 anos de escolaridade e de $11,1 \%$ para os dois grupos de escolaridade intermediária, 5 a 8 e 9 a 11 anos ${ }^{21}$.

Quando perguntados sobre os serviços procurados quando se sentiram doentes ou quando necessitaram fazer uma consulta de saúde, no período de 12 meses anteriores à entrevista, uma maior proporção dos idosos com até 4 anos de escolaridade-33,6\% indicou hospital público, seguida de clínica pública, com $29,7 \%$, mostrando claramente os serviços públicos como sua principal alternativa e, além disso, o acesso direto a serviços hospitalares. Uma proporção de 25,1\% deles procurou consultório privado e, 15,1\%, hospital particular.

Nos demais grupos de escolaridade o tipo de serviço mais procurado foi o Consultório Privado, seguido de Hospital Particular, conforme pode ser visto na Tabela 10. O grupo de escolaridade mais alta, embora tenha feito uma procura maior de serviços privados, tanto consultório, com $63,2 \%$, como hospitalar, com $25,1 \%$, referiu também a procura por Hospital Público - 19,5\% - de forma distinta dos grupos de escolaridade intermediária, onde essa procura foi de $13,5 \%$ e $11 \%$, respectivamente, para os grupos de escolaridade 5 a 8 anos e 9 a 11 anos.

A freqüência dos que responderam não terem adoecido no período de 12 meses e nem procurado serviços de saúde variou de $13,5 \%$ no grupo com escolaridade até 4 anos a 19,9\% no grupo de 5 a 8 anos, e apresentou valores intermediários nos dois outros grupos. Poucos entrevistados referiram outras alternativas para resolverem seus problemas 
Tabela 10 - Tipo de serviço de saúde procurado para morbidade de 12 meses segundo escolaridade, São Paulo, 2000. Table 10 - Type of health service for 12-month morbidity, by years of education, São Paulo, 2000.

\begin{tabular}{lcccc}
\hline Serviço de Saúde Procurado & \multicolumn{3}{c}{ Anos de Escolaridade } \\
& até 4 & 5 a 8 & 9 a 11 & 12 ou mais \\
\hline Consultório. Particular & 25,1 & 46,0 & 52,8 & 63,2 \\
Clínica pública & 29,7 & 19,9 & 6,5 & 0,0 \\
Hospital. Particular $^{1}$ & 15,1 & 18,6 & 33,7 & 25,1 \\
Hospital. Público $^{1}$ & 33,6 & 13,5 & 11,0 & 19,5 \\
Não ficou doente & 13,5 & 19,9 & 15,4 & 14,6 \\
Outros, não procurou $^{2}$ & 4,8 & 2,5 & 1,3 & 0,5 \\
\hline
\end{tabular}

1 - Emergência de Hospital ou Hospital

2 - Inclui Farmácia, Curandeiros, Outros e os que não procuraram serviços de saúde

de saúde, como farmácias ou curandeiros, refletindo o padrão de uso de serviços de saúde de área urbana e industrializada, mesmo com uma grande parcela da população vivendo em condições precárias e com dificuldades de acesso aos serviços ${ }^{21}$.

\section{Renda e condição de emprego}

Os dados relativos à condição de emprego e à renda revelam, em primeiro lugar, que a grande maioria dos idosos sempre desenvolveu alguma atividade e, de modo preponderante, uma atividade remunerada. Em segundo lugar, uma parcela não desprezível ainda continuava trabalhando à época da pesquisa, justificando esse fato pela necessidade de ter remuneração.

No que diz respeito à renda total constatou-se nível relativamente baixo, 2,1 salários mínimos em média. Um aspecto que chama a atenção é o fato de que grande parte dos idosos era responsável por um grupo familiar, pois a renda per capita é apenas $44 \%$ da renda média total. No entanto, $47 \%$ não tinham renda monetária alguma. Desconsiderando-se esse segmento dos idosos, a renda média daqueles que tinham renda era bem superior, da ordem de 5,5 salários mínimos ${ }^{22}$.

Tomando-se como indicador da escolaridade a freqüência à escola, verifica-se que o rendimento dos idosos com algum grau de escolaridade é superior em quase $114 \%$ ao dos que não tiveram essa oportunidade, enquanto a proporção dos sem renda é semelhante entre os dois grupos e varia entre
$50 \%$ para os que tiveram alguma educação e $58,5 \%$ para os demais.

Além disso, verificou-se que o perfil da distribuição de renda entre os idosos repete o perfil da distribuição em nível nacional, ligeiramente melhorada, mas ainda uma distribuição fortemente concentrada, onde, considerando-se inclusive os que não têm renda, os $40 \%$ mais pobres respondiam por apenas $9,5 \%$ da renda dos idosos e os $10 \%$ mais ricos por $48 \%$. Um índice de Gini de 0,54 está associado a essa distribuição, um pouco abaixo do índice de concentração da distribuição de renda nacional, que foi de 0,567 para 1999, conforme o IBGE.

Esse fato sugere que, entre essa população, há segmentos com níveis de rendimento insuficientes para uma vida digna ao final do ciclo vital; na realidade, mais de $50 \%$ dos idosos relataram não ter renda. Como esperado, as mulheres auferiam níveis de renda inferiores aos dos homens, enquanto os indivíduos das duas primeiras faixas de idade tinham, em média, maior renda do que os da última faixa.

As rendas provenientes da remuneração do trabalho apareceram como a principal fonte, seguida dos aluguéis ${ }^{22}$.

\section{Conclusão}

Pode-se dizer que é preocupante a avaliação do estado de saúde nos níveis encontrados, pois isso provavelmente está diretamente relacionado a uma baixa qualidade de vida, influenciada pelo gênero, escolaridade, 
idade, condição econômica e presença de incapacidade. Essa realidade deve alertar os planejadores de saúde a fim de ser adequada a oferta de serviços à demanda representada pelos idosos de hoje e os que virão.

O sistema de seguridade social não tem se constituído na principal instituição a garantir a subsistência da população que já contribuiu ao longo de sua vida para a produção social do país, pois grande parcela dessa população depende ainda de remuneração do trabalho e do patrimônio que acumulou. Considerando que a época atual vem se caracterizando pelo aumento do desemprego, é de se esperar que os idosos futuros, aqueles que em 10 ou 20 anos estarão acima dos 60 anos, não terão essas duas principais fontes de renda para complementar, e mesmo garantir, a sua sobrevivência e, assim, o aumento da expectativa de vida alcançado graças aos avanços da medicina não estarão associados a maior bem-estar social.

Atualmente, a questão preponderante em relação à velhice está, portanto, relacionada ao equilíbrio entre as necessidades e as exigências mínimas dos idosos e a capacidade assistencial disponível para atendê-las, em nível familiar ou coletivo, pois, nessa fase da vida, tais aspectos adquirem características especiais.

\section{Agradecimentos}

À Dra Martha Peláez, pela coordenação competente e afetuosa do Projeto SABE, que juntamente com Prof. Dr. Alberto Palloni tornaram esse Estudo realidade.

Ao Sr. Fernão Dias de Lima, pela competente administração da base de dados e posteriores tabulações.

\section{Referências}

1. Palloni A, Peláez M. Histórico e natureza do estudo. In: Lebrão, ML; Duarte, YAO (org). O Projeto SABE no Município de São Paulo: uma abordagem inicial. Brasília: OPAS/MS; 2003. p.15-32.

2. Silva NN. Aspectos metodológicos: processo de amostragem. In: Lebrão, ML; Duarte, YAO (org). $O$ Projeto SABE no Município de São Paulo: uma abordagem inicial. Brasília: OPAS/MS; 2003. p. 47-57.

3. Lebrão ML, Duarte YAO (org). O Projeto SABE no Município de São Paulo: uma abordagem inicial. Brasília: OPAS/MS; 2003.

4. Icaza MC, Albala C. Projeto SABE. Minimental State Examination (MMSE) del estudio de dementia en Chile: análisis estatístico. OPAS; 1999: 1-18.

5. Folstein MF, Folstein SE, McHugh PR. A practical method for grading the cognitive state of patient for the clinician. J Psychiatr Res 1975; 12: 189-98.

6. Pfeffer RI, Kurosaki TT, Harrah Jr CH, Chance JM, Filos RN. Measurement of functional activities in older adults in the community. J Gerontol 1987; 37: 323-9.

7. Sheikh JI, Yesavage JA. Geriatric Depression Scale (GDS): recent evidence and development of a short version. Clin Gerontol 1986; 5: 165-73.

8. Ramos LR, Rosa TEC, Oliveira ZM, Medina MCG, Santos FRG. Perfil do idoso em área metropolitana na região sudeste do Brasil: resultados de inquérito domiciliar. Rev Saúde Pública 1993; 27(2): 87-94.
9. Cerqueira ATAR. Deterioração cognitiva e depressão. In: Lebrão, ML; Duarte, YAO (org). O Projeto SABE no Município de São Paulo: uma abordagem inicial. Brasília: OPAS/MS; 2003. p. 143-65.

10. Idler EL, Benyamini Y. Self rated health and mortality: a review of twenty-seven community studies. $J$ Health Soc Behav 1997; 38: 21-37.

11. Lebrão, ML; Laurenti, R. Condições de saúde. In: Lebrão, ML; Duarte, YAO (org). O Projeto SABE no Município de São Paulo: uma abordagem inicial. Brasília: OPAS/MS; 2003. p. 75-91.

12. Santos JLF. Análise da sobrevida sem incapacidades. In: Lebrão, ML; Duarte, YAO (org). O Projeto SABE no Município de São Paulo: uma abordagem inicial. Brasília: OPAS/MS; 2003. p. 169-81.

13. Narvai PC; Antunes JLF. Saúde bucal: a autopercepção da mutilação e das incapacidades. In: Lebrão, ML; Duarte, YAO (org). O Projeto SABE no Município de São Paulo: uma abordagem inicial. Brasília: OPAS/MS, 2003; p. 121-40.

14. Marucci MFN, Barbosa AR. Estado nutricional e capacidade física. In: Lebrão, ML; Duarte, YAO (org). $O$ Projeto SABE no Município de São Paulo: uma abordagem inicial. Brasília: OPAS/MS; 2003. p. 95-117.

15. Saad, PM. Transferencias informales de apoyo de los adultos mayores em América Latina y el Caribe: estudio comparativo de encuestas. SABE. Notas población. 2003; 3(77): 175-217. 
16. Duarte YAO, Lebrão ML, Lima FD. Contribuição dos arranjos domiciliares para o suprimento de demandas assistenciais dos idosos com comprometimento funcional. São Paulo, Brasil; 2004 [aceito para publicação na Revista Panamericana de Salud Pública]

17. Medeiros, M; Osório, R. Arranjos domiciliares e arranjos nucleares no Brasil: classificação e evolução de 1977 a 1998. Brasília: IPEA; 2001.

18. Saad, PM. Transferências informales de apoyo de los adultos mayores em América Latina y el Caribe: estúdio comparativo de encuestas. SABE; [no prelo], 2004 .

19. Hays, JC. Living arrangements and health status in later life: a review of recent literature. Public Health Nurs 2002; 199(2): 136-51.
20. Ebersole, P; Hess, P. Relationships and their significance. In: Ebersole, P; Hess,P. Toward healthy aging: human needs and nursing response. 5a.ed, S.Louis: Mosby; 1998. p. 606-39.

21. César, CLG, Paschoal, SMP. Uso dos serviços de saúde. In: Lebrão, ML; Duarte, YAO (org). O Projeto SABE no Município de São Paulo: uma abordagem inicial. Brasília: OPAS/MS; 2003. p. 227-37.

22. Campino ACC, Cyrillo DC. Situação de ocupação e renda. In: Lebrão, ML; Duarte, YAO (org). O Projeto SABE no Município de São Paulo: uma abordagem inicial. Brasília: OPAS/MS; 2003. p. 241-55.

recebido em: 15/12/04 versão final reapresentada em: 25/05/05 aprovado em: 27/05/05 